\title{
THE ALGEBRA OF DIFFERENTIAL OPERATORS FOR A MATRIX WEIGHT. AN ULTRASPHERICAL EXAMPLE.
}

\author{
IGNACIO N. ZURRIÁN
}

\begin{abstract}
In this paper we study in detail algebraic properties of the algebra $\mathcal{D}(W)$ of differential operators associated to a matrix weight of Gegenbauer type. We prove that two second order operators generate the algebra, indeed $\mathcal{D}(W)$ is isomorphic to the free algebra generated by two elements subject to certain relations. Also, the center is isomorphic to the affine algebra of a singular rational curve. The algebra $\mathcal{D}(W)$ is a finitely-generated torsion-free module over its center, but it is not flat and therefore it is not projective.

This is the second detailed study of an algebra $\mathcal{D}(W)$ and the first one coming from spherical functions and group representations. We prove that the algebras for different Gegenbauer weights and the algebras studied previously, related to Hermite weights, are isomorphic to each other. We give some general results that allow us to regard the algebra $\mathcal{D}(W)$ as the centralizer of its center in the Weyl algebra. We do believe that this should hold for any irreducible weight and the case considered in this paper represents a good step in this direction.
\end{abstract}

\section{INTRODUCTION}

The subject of matrix-valued orthogonal polynomials was introduced by M.G. Krein more than sixty years ago, see Kre49, Kre71. In the scalar valued context, the orthogonal polynomial satisfying second order differential equations played a very important role in many areas of mathematics and its applications. A.J. Durán started the study of matrix-weights whose inner product admits a symmetric second order matrix differential operator in Dur97, following similar considerations by S. Bochner in the scalar case in Boc29. A general approach to the case of higher order can be found in [DG86, GH97.

Motivated by J.A. Tirao's work on the theory of matrix-valued spherical functions, see [Tir77, one finds in GPT02a, GPT03, Grü03 the first examples of situations as those considered in Dur97; in DG04 one can find the development of a general method that leads to introduce and study classes of examples of orthonormal matrix polynomials. In the last years, one can see a growing number of papers devoted to different aspects of this question. For some of these recent papers, see GPT01, GPT02b, Tir03, GPT04, DG05a, DG05c, DG05b, DG05d, CG05, GPT05, Mir05, PT07) as well as DdlI08a, DdlI08b, PR08, GdlIMF11, dlI11, KvPR12, KvPR13, CdlI14, vPR14, AKdlR15.

For a given matrix weight $W(x)$ of size $N$ on the real line, on can consider a sequence of matrix orthogonal polynomials with respect to $W$. Then, it is natural to think in the algebra $\mathcal{D}(W)$ of matrix differential operators having as eigenfunctions all these polynomials. A first attempt to go beyond the existence of nontrivial elements in $\mathcal{D}(W)$ and to study the full algebra (experimentally, with the assistance of symbolic computation) is undertaken in CG06. In Tir11 we can find a deep study of an algebra $\mathcal{D}(W)$. In that case the author considered the simplest possible example, which was one of the five examples included earlier in CG06.

2010 Mathematics Subject Classification. 13N10; 16S32; 33C45; 35P05.

Key words and phrases. Matrix Orthogonal Polynomials, Matrix Differential Operators, Bispectral Problem, Differential Operators Algebra.

This research was supported through the programme "Oberwolfach Leibniz Fellows" by the Mathematisches Forschungsinstitut Oberwolfach in 2015 and CONICET Argentina. 
We wish to understand some algebraic properties of the algebra $\mathcal{D}(W)$ and in this work we consider an example coming from group representation theory. More precisely, our weight $W$ and its orthogonal polynomials were studied in [PZ15] based on [TZ14]. Namely,

$$
W(x)=W_{p, n}=\left(1-x^{2}\right)^{\frac{n}{2}-1}\left(\begin{array}{cc}
p x^{2}+n-p & -n x \\
-n x & (n-p) x^{2}+p
\end{array}\right), \quad x \in[-1,1],
$$

for real parameters $0<p<n / 2$. This was the example employed in the study of the first case of the time and band limiting problem for matrix-valued functions (see GPZ15]).

This algebra is of interest by itself. Even though it is not commutative and in the context of bispectrality one of the variables is discrete, there are strong motivations coming from the classical theory of commuting operators. Among all the equations that are considered in the framework of the inverse scattering method we can mention the Korteweg-de Vries equation and its two dimensional generalization, the Kadomtsev-Petviashvili equation, the non-linear Schrödinger equation, the sine-Gordon equation and many other fundamental equations of modern mathematical physics. All these equations can be represented as compatibility conditions for an overdetermined system of auxiliary linear problems. For example, for the $\mathrm{KdV}$ equation, this system has the form

$$
D \psi=0, \quad \psi_{t}=E \psi,
$$

with

$$
D=-\frac{\partial^{2}}{\partial x^{2}}+u(x, t), \quad E=\frac{\partial^{3}}{\partial x^{3}}-\frac{3}{2} u \frac{\partial}{\partial x}-\frac{3}{4} u_{x} .
$$

The compatibility of this system implies

$$
\left[\frac{\partial}{\partial t}-E, D\right]=0 \quad \Longleftrightarrow \quad D_{t}=[E, D] .
$$

This operator equation is called "Lax equation"; each Lax equation is an infinite-dimensional analogue of the completely integrable systems. For the KdV equation, Novikov considered the restriction to the space of stationary solutions, which is essentially equivalent to consider a particular case of the more general problem of the classification of commuting ordinary differential operators with scalar coefficients. As a pure algebraic problem it was considered by Burchnall and Chaundy in [BC23, BC28]; given two commuting operators $D$ and $E$ of order $s$ and $\ell$, respectively, there exists a polynomial $R(\lambda, \mu)$ in two variables such that

$$
R(D, E)=0 \text {. }
$$

If $s$ and $\ell$ are coprime then for each point $q=(\lambda, \mu)$ of the curve $\Gamma$, that is defined in $\mathbb{C}^{2}$ by the equation $R(\lambda, \mu)=0$, there corresponds a unique (up to constant factor) common eigenfunction $\psi(x, q)$ of $D$ and $E$ :

$$
D \psi(x, q)=\lambda \psi(x, q), \quad E \psi(x, q)=\mu \psi(x, q) .
$$

These commuting pairs are classified by a set of algebro-geometric data, see [Kri81, Mum77, Tak05.

Of course, working in a scalar context is crucial, as one would expect; if we are dealing with a noncommutative ring of coefficients, such as the ring of matrices, things are more complicated. By considering only commuting differential operators of order two one has a very illuminating contrast. In the scalar case the coefficients of the highest derivatives of the commuting operators have to be the same up to a constant factor, thus one is essentially dealing with an operator of order two and another of order one. In the matrix case this is more complicated. In [KV01] one can find an interesting discussion and results on commuting matrix differential operators. However, a natural source is the algebra $\mathcal{D}(W)$, with special attention to its center or even to the abelian maximal subalgebras. Particularly, we prove that our algebra $\mathcal{D}(W)$ is the centralizer of its center in the Weyl algebra $\mathbf{D}$ given by

$$
\mathbf{D}=\left\{D=\sum_{i=0}^{s} \partial^{i} F_{i}: F_{i} \in M_{N}[x]\right\}
$$


We prove that for any weight $W$, under certain conditions, this presentation of the algebra in terms of its center is still valid. We expect that this presentation is always possible for any irreducible weight.

Now, we proceed to describe the contents of each section. In Section 2 we give a brief review of some general notions and properties of the algebras $\mathcal{D}(W)$.

In Section 3 we introduce our Gegenbauer example. We prove that our weights $W_{p, n}, 0<p<n / 2$, are non-similar each other. Even though this weight is very different from the weight considered in [Tir11], we take advantage of some ingenious tools used by Tirao to simplify some of the conditions satisfied by the operators in $\mathcal{D}(W)$ and, at the beginning, we follow the line of argument in Tir11.

In Section 4 we prove that for any operator

$$
D=\sum_{\ell=0}^{s} \partial^{\ell} F_{\ell}(x) \in \mathcal{D}(W)
$$

if $F_{s} \neq \mathbf{0}$ then $\operatorname{deg}\left(F_{s}\right)=s$ and $s$ is even. Even more, we have four operators $D_{1}, D_{2}, D_{3}, D_{4}$ of order two that generate the algebra $\mathcal{D}(W)$.

In Section 5 we prove that $\mathcal{D}(W)$ is isomorphic to the free algebra generated by two elements, $A$ and $B$, with the following relations

$$
\begin{aligned}
B^{2} A-A B^{2} & =0, & B A^{2}+A^{2} B-2 A B A-B & =0, \\
B A B+A^{3}-2 A B^{2}-A & =0, & B^{3}-2 A^{2} B+A B A & =0 .
\end{aligned}
$$

We also prove that all these non-similar weights $W_{p, n}$ have isomorphic algebras $\mathcal{D}\left(W_{p, n}\right)$.

Also, in spite of the fact that the operators in [Tir11] are clearly different to the operators here, we prove that the algebras are isomorphic.

In Section 6 we focus our attention on the center $\mathcal{Z}(W)$ of the algebra $\mathcal{D}(W)$, proving that it is generated by two operators $C_{1}$ and $C_{2}$ of order four and six, respectively. Even more, the center of the algebra $\mathcal{D}\left(W_{p, n}\right)$ is isomorphic to the affine algebra of the following singular rational curve:

$$
x^{3}-y^{2}=(n-2 p) x y \text {. }
$$

In the process of determining whether this is an Azumaya algebra or not, we prove that the algebra $\mathcal{D}(W)$ is a finitely-generated torsion-free module over the ring $\mathcal{Z}(W)$, but it is not flat and therefore not projective.

In Section 7 we prove that under certain conditions, the algebra $\mathcal{D}(W)$ is the centralizer of its center in the Weyl algebra.

We are convinced that the study of these algebras can be useful for a better understanding of the theory of matrix orthogonal polynomials that satisfy differential equations of order two. There is recent work [TZ15, which includes very simple and direct criteria for the reducibility of a matrix weight $W$ based on the algebra $\mathcal{D}(W)$. After carrying out the comparison and deep study of these algebras, we believe that a natural step is to start a classification of matrix differential operators associated with matrix weights, aiming at giving a description of the matrix commuting operators in algebro-geometric terms.

\section{Preliminaries}

Let $W=W(x)$ be a weight matrix of size $N \in \mathbb{N}$ on the real line. By this we mean a complex $N \times N$ matrixvalued integrable function on the interval $(a, b)$ such that $W(x)$ is positive definitive almost everywhere and with finite moments of all orders. From now on we shall denote by $M_{N}$ the algebra of all $N \times N$ matrices over $\mathbb{C}$ and by $M_{N}[x]$ the algebra over $\mathbb{C}$ of all polynomials in $x$ with coefficients in $M_{N}$. We denote $\mathbf{I}$ the identity of $M_{N}$ and by $T^{*}$ the transpose conjugate of $T$. We introduce as in Kre49 and Kre71 the following matrix-valued Hermitian sesquilinear form in the linear space $M_{N}[x]$ :

$$
(P, Q)=\int_{a}^{b} P(x) W(x) Q(x)^{*} d x .
$$


Then $M_{N}[x]$ is a left inner product $M_{N}$-module and there exists a unique sequence $\left\{Q_{w}\right\}_{w \geq 0}$ of monic orthogonal polynomials. More generally, a sequence $\left\{P_{w}\right\}_{w \geq 0}$ of matrix orthogonal polynomials is a sequence of elements $P_{w} \in M_{N}[x]$ such that $P_{w}$ is of degree $w$, its leading coefficient is a non singular matrix and $\left(P_{w}^{\prime}, P_{w}\right)=0$ for all $w^{\prime} \neq w$. Then any sequence $\left\{P_{w}\right\}_{w>0}$ of matrix orthogonal polynomials is of the form $P_{w}=A_{w} Q_{w}$ where $A_{w} \in G L_{N}(\mathbb{C})$ is arbitrary for each $w \geq 0$. We come now to the notion of a differential operator with matrix coefficients acting on matrix-valued polynomials, i.e. elements of $M_{N}[x]$. These operators can be made to act on our functions either on the left or on the right (see [Dur97]), but it turns out to be convenient to work with differential operators acting on the right. Then, $D$ given by

$$
D=\sum_{i=0}^{s} \partial^{i} F_{i}(x), \quad \partial=\frac{d}{d x}
$$

acts on $P(x)$ by means of

$$
P D(x)=\sum_{i=0}^{s} \partial^{i}(P)(x) F_{i}(x), \quad \partial=\frac{d}{d x} .
$$

Notice that if $D_{1}$ and $D_{2}$ are two differential operators we have $P\left(D_{1} D_{2}\right)=\left(P D_{1}\right) D_{2}$. The following three propositions are taken from GT07.

Proposition 2.1 (GT07, Proposition 2.6). Let $W=W(x)$ be a weight matrix of size $N$ and let $\left\{Q_{w}\right\}_{w \geq 0}$ be the sequence of monic orthogonal polynomials in $M_{N}[x]$. If

$$
D=\sum_{i=0}^{s} \partial^{i} F_{i}(x), \quad \partial=\frac{d}{d x},
$$

is a linear right-hand side ordinary differential operator of order s such that

$$
Q_{w} D=\Lambda_{w} Q_{w}, \quad \text { for all } \quad w \geq 0,
$$

with $\Lambda_{w} \in M_{N}$, then $F_{i}=F_{i}(x) \in M_{N}[x]$ and $\operatorname{deg} F_{i} \leq i$. Moreover $D$ is determined by the sequence $\left\{\Lambda_{w}\right\}_{w \geq 0}$

We could have written the eigenvalue matrix $\Lambda_{w}$ to the right of the matrix valued polynomials $Q_{w}$ above. However, as shown in Dur97, this only leads to uninteresting cases where the weight matrix is diagonal. To ease the notation, if $\nu \in \mathbb{C}$ let

$$
[\nu]_{i}=\nu(\nu-1) \cdots(\nu-i+1), \quad[\nu]_{0}=1 .
$$

Proposition 2.2 (GT07, Proposition 2.7). Let $D=\sum_{i=0}^{s} \partial^{i} F_{i}(x)$ satisfy (1), with

$$
F_{i}(x)=\sum_{j=0}^{i} F_{j}^{i}(x) .
$$

Then

$$
\Lambda_{w}=\sum_{j=0}^{s}[w]_{i} F_{i}^{i} \quad \text { for all } \quad w \geq 0 .
$$

Hence, $w \rightarrow \Lambda_{w}$ is a matrix-valued polynomial function with $\operatorname{deg} \Lambda_{w} \leq \operatorname{deg}(D)$.

Given a sequence of matrix orthogonal polynomials $\left\{Q_{w}\right\}_{w \geq 0}$ we are interested in the algebra $\mathcal{D}(W)$ of all right-hand side differential operators with matrix-valued coefficients that have the polynomials $Q_{w}$ as eigenfunctions. Notice that if $Q_{w} D=\Lambda_{w} Q_{w}$ for some eigenvalue matrix $\Lambda_{w} \in M_{N}$, then $\Lambda_{w}$ is uniquely determined by $D$. In such a case we write $\Lambda_{w}(D)=\Lambda_{w}$. Thus 


$$
\mathcal{D}(W)=\left\{D: Q_{w} D=\Lambda_{w}(D) Q_{w}, \Lambda_{w}(D) \in M_{N} \text { for all } w \geq 0\right\} .
$$

First of all we observe that the definition of $\mathcal{D}(W)$ depends only on the weight matrix $W=W(x)$ and not on the sequence $\left\{Q_{w}\right\}_{w \geq 0}$.

Proposition 2.3 (GT07, Proposition 2.8). Given a sequence $\left\{Q_{w}\right\}_{w \geq 0}$ of orthogonal polynomials let the algebra $\mathcal{D}(W)$ be as in (3). Then $D \rightarrow \Lambda_{w}(D)$ is a representation of $\mathcal{D}(W)$ into $M_{N}$, for each $w \geq 0$. Moreover the sequence of representations $\left\{\Lambda_{w}\right\}_{w \geq 0}$ separates the elements of $\mathcal{D}(W)$.

In particular, if $\left\{Q_{w}\right\}_{w \geq 0}$ is the sequence of monic orthogonal polynomials we have a homomorphism

$$
\Delta=\prod \Delta_{w}: \mathcal{D}(W) \rightarrow M_{N} \mathbb{N}_{0}
$$

of $\mathcal{D}(W)$ into the direct product of $\mathbb{N}_{0}$ copies of $M_{N}$. Moreover $\Delta$ is injective.

In Section 3 of GT07, the ad-conditions coming from the bispectral pairs $(L, D)$ are used to described the image $\Delta(W)$ of $\mathcal{D}(W)$ by the eigenvalue isomorphism $\Delta$. Here $L$ is the difference operator associated to the three term recursion relation satisfied by the sequence of monic orthogonal polynomials and $D \in \mathcal{D}(W)$. This gives a completely different presentation of $\mathcal{D}(W)$ and will be used frequently to simplify several computations. Let us mention that the ad-conditions were first introduced in [DG86].

\section{The $2 \times 2$ Gegenbauer Example}

From now on we will consider the matrix-valued orthogonal polynomials related to the $2 \times 2$ weight matrix given by

$$
W(x)=W_{p, n}=\left(1-x^{2}\right)^{\frac{n}{2}-1}\left(\begin{array}{cc}
p x^{2}+n-p & -n x \\
-n x & (n-p) x^{2}+p
\end{array}\right), \quad x \in[-1,1],
$$

for real parameters $0<p<n / 2$.

The orthogonal polynomials arising here are discussed in full in [PZ15. As we said in the introduction, the purpose of this paper is to compute in this case the algebra $\mathcal{D}(W)$ and to study its structure. The monic orthogonal polynomials $\left\{Q_{w}\right\}_{w \geq 0}$ with respect to the weight matrix $W(x)$ are explicitly given by

$$
Q_{w}=\frac{w !}{2^{w}\left(\frac{n+1}{2}\right)_{w}}\left(\begin{array}{cc}
C_{w}^{\frac{n+1}{2}}(x)+\frac{n+1}{p+w} C_{w-2}^{\frac{n+3}{2}}(x) & \frac{n+1}{p+w} C_{w-1}^{\frac{n+3}{2}}(x) \\
\frac{n+1}{n-p+w} C_{w-1}^{\frac{n+3}{2}}(x) & C_{w}^{\frac{n+1}{2}}(x)+\frac{n+1}{n-p+w} C_{w-2}^{\frac{n+3}{2}}(x)
\end{array}\right),
$$

where $(a)_{w}=a(a+1) \ldots(a+w-1)$ denotes the Pochhammer's symbol and $C_{n}^{\lambda}(x)$ denotes the $n$-th Gegenbauer polynomial

$$
C_{n}^{\lambda}(x)=\frac{(2 \lambda)_{n}}{n !}{ }_{2} F_{1}\left(\begin{array}{c}
-n, n+2 \lambda \\
\lambda+1 / 2
\end{array} ; \frac{1-x}{2}\right) .
$$

As usual, we assume $C_{n}^{\lambda}(x)=0$ if $n<0$; recall that $C_{n}^{\lambda}$ is a polynomial of degree $n$, with leading coefficient $\frac{2^{n}(\lambda)_{n}}{n !}$. Recall that (see [AS65, p 561])

$$
C_{n}^{(\alpha)}(z)=\sum_{k=0}^{\lfloor n / 2\rfloor}(-1)^{k} \frac{\Gamma(n-k+\alpha)}{\Gamma(\alpha) k !(n-2 k) !}(2 z)^{n-2 k} .
$$

Let us recall the concept of similarity for matrix weights. Two weights $W$ and $\widetilde{W}$ are said to be similar if there exists a nonsingular matrix $M$, which does not depend on $x$, such that

$$
\widetilde{W}(x)=M W(x) M^{*}, \quad \text { for all } x \in(a, b) .
$$

Lemma 3.1. If $(p, n) \neq(\tilde{p}, \tilde{n})$ then $W_{p, n}$ is not similar to $W_{\tilde{p}, \tilde{n}}$. 
Proof. If $W_{p, n}$ is similar to $W_{\tilde{p}, \tilde{n}}$ then there exists a nonsingular matrix $M=\left(\begin{array}{ll}a & b \\ c & d\end{array}\right)$ such that $\widetilde{W}=M W M *$, therefore it is clear that $n=\tilde{n}$. Hence we have that the matrix $\left(\begin{array}{c}\tilde{p} x^{2}+n-\tilde{p}-n x \\ -n x(n-\tilde{p}) x^{2}+\tilde{p}\end{array}\right)$ is of the form

$$
\left(\begin{array}{cc}
\left(|a|^{2} p+|b|^{2} n-|b|^{2} p\right) x^{2}+(-\bar{a} b n-\bar{b} a n) x+|a|^{2} n-|a|^{2} p+|b|^{2} p & (\bar{c} a p+(b n-b p) \bar{d}) x^{2}+(-b n \bar{c}-a n \bar{d}) x+(a n-a p) \bar{c}+\bar{d} b p \\
(\bar{a} c p+(d n-d p) \bar{b}) x^{2}+(-d n \bar{a}-c n \bar{b}) x+(c n-c p) \bar{a}+\bar{b} d p & \left(|c|^{2} p+|d|^{2} n-|d|^{2} p\right) x^{2}+(-\bar{c} d n-\bar{d} c n) x+|c|^{2} n-|c|^{2} p+|d|^{2} p
\end{array}\right) .
$$

Then, by looking at the entry $(1,2)$ in the matrix above, we have

$$
(\bar{c} a p+(b n-b p) \bar{d})=0 \quad \text { and } \quad(a n-a p) \bar{c}+\bar{d} b p=0,
$$

but since $M$ is invertible and $0<p<n / 2$ we have that $M$ has to be the identity matrix. Hence $(p, n)=$ $(\tilde{p}, \tilde{n})$.

Let us denote

$$
Q_{w}=\sum_{i=0}^{w} x^{i} B_{i}^{w}
$$

From (5) and (6) we obtain

$$
\begin{aligned}
& B_{w-2 k}^{w}=\frac{w !(-1)^{k} 2^{-2 k}}{\left(\frac{n+1}{2}+w-k\right)_{k} k !(w-2 k) !}\left(\begin{array}{cc}
\frac{p+w-2 k}{p+w} & 0 \\
0 & \frac{n-p+w-2 k}{n-p+w}
\end{array}\right), \\
& B_{w-2 k-1}^{w}=\frac{w !(-1)^{k} 2^{-2 k}}{\left(\frac{n+1}{2}+w-k\right)_{k} k !(w-2 k-1) !}\left(\begin{array}{cc}
0 & \frac{1}{p+w} \\
\frac{1}{n-p+w} & 0
\end{array}\right) .
\end{aligned}
$$

We will use some tools already employed by Tirao to simplify some of the conditions satisfied by the operators in $\mathcal{D}(W)$. We quote a result in [Tir11] (see Proposition 3.4 and equation (25)), making some changes in the parameters.

Proposition 3.2. Let $D=\sum_{j=0}^{s} \partial F_{j}(x)$, with $F_{j}=\sum_{i=0}^{j} x^{i} F_{i}^{j}$. Then $D \in \mathcal{D}(W)$ if and only if

$$
\sum_{i=0}^{s} B_{w-m+i}^{w}\left(\sum_{\ell=0}^{s}[w-m+i]_{\ell} F_{\ell-i}^{\ell}\right)-\left(\sum_{\ell=0}^{s}[w]_{\ell} F_{\ell}^{\ell}\right) B_{w-m}^{w}=0
$$

for all $0 \leq m \leq w, 0 \leq w$.

The following lemma states a nice property of the weight $W$ that can be easily proved.

Lemma 3.3. Given

$$
T=\left(\begin{array}{cc}
1 & 0 \\
0 & -1
\end{array}\right)
$$

we have that

$$
W(-x)=T W(x) T .
$$

Definition 3.4. Given $D=\sum_{0 \leq i \leq s} \partial^{i} F_{i} \in \mathcal{D}$ let

$$
\widetilde{D}=\sum_{0 \leq i \leq s} \partial^{i}(-1)^{i} T \widetilde{F}_{i} T \in \mathcal{D},
$$

where $\widetilde{F}_{i}(x)=F_{i}(-x)$. 
THE ALGEBRA OF DIFFERENTIAL OPERATORS FOR A MATRIX WEIGHT. AN ULTRASPHERICAL EXAMPLE. 7

Proposition 3.5 (Tir11], Proposition 3.9). If $D \in \mathcal{D}(W)$ then $\widetilde{D} \in \mathcal{D}(W)$. Moreover, if $\left\{Q_{w}\right\}_{w \geq 0}$ is the sequence of monic orthogonal polynomials with respect to $W$ and $\left\{\Lambda_{w}(D)\right\}_{w \geq 0}$ is the corresponding eigenvalue sequence, then

$$
\Lambda_{w}(\widetilde{D})=T \Lambda_{w}(D) T
$$

Theorem 3.6 (Tir11], Theorem 3.10). The map $D \rightarrow \widetilde{D}$ defines an involutive automorphism of the algebra $\mathcal{D}(W)$.

We will need the following lemma from [PZ15].

Lemma 3.7 (PZ15, Corollary 5.3). The set of differential operators of order at most two in $\mathcal{D}(W)$ is a five-dimensional vector space generated by the identity $\mathbf{I}$ and

$$
\begin{aligned}
& D_{1}=\partial^{2}\left(\begin{array}{cc}
x^{2} & x \\
-x & -1
\end{array}\right)+\partial\left(\begin{array}{cc}
(n+2) x & n-p+2 \\
-p & 0
\end{array}\right)+\left(\begin{array}{cc}
p(n-p+1) & 0 \\
0 & 0
\end{array}\right), \\
& D_{2}=\partial^{2}\left(\begin{array}{cc}
-1 & -x \\
x & x^{2}
\end{array}\right)+\partial\left(\begin{array}{cc}
0 & p-n \\
p+2 & (n+2) x
\end{array}\right)+\left(\begin{array}{cc}
0 & 0 \\
0 & (p+1)(n-p)
\end{array}\right), \\
& D_{3}=\partial^{2}\left(\begin{array}{cc}
-x & -1 \\
x^{2} & x
\end{array}\right)+\partial\left(\begin{array}{cc}
-p & 0 \\
2(p+1) x & p+2
\end{array}\right)+\left(\begin{array}{cc}
0 & 0 \\
p(p+1) & 0
\end{array}\right), \\
& D_{4}=\partial^{2}\left(\begin{array}{cc}
x & x^{2} \\
-1 & -x
\end{array}\right)+\partial\left(\begin{array}{cc}
n-p+2 & 2(n-p+1) x \\
0 & p-n
\end{array}\right)+\left(\begin{array}{cc}
0 & (n-p)(n-p+1) \\
0 & 0
\end{array}\right) .
\end{aligned}
$$

The corresponding eigenvalues are given by

$$
\begin{array}{ll}
\Lambda_{w}\left(D_{1}\right)=\left(\begin{array}{cc}
(w+p)(w+n-p+1) & 0 \\
0 & 0
\end{array}\right), & \Lambda_{w}\left(D_{2}\right)=\left(\begin{array}{cc}
0 & 0 \\
0 & (w+p+1)(w+n-p)
\end{array}\right), \\
\Lambda_{w}\left(D_{3}\right)=\left(\begin{array}{cc}
0 \\
(w+p)(w+p+1) & 0
\end{array}\right), & \Lambda_{w}\left(D_{4}\right)=\left(\begin{array}{c}
0(w+n-p)(w+n-p+1) \\
0
\end{array}\right) .
\end{array}
$$

It can be easily checked that

$$
\widetilde{D}_{1}=D_{1}, \quad \widetilde{D}_{2}=D_{2}, \quad \widetilde{D}_{3}=-D_{3} \quad \widetilde{D}_{4}=-D_{4},
$$

It is worthwhile paying attention to the expression of the matrix eigenvalues in Lemma 3.7. They will be used in some proofs and they imply the following additional remark.

Remark 3.8. We have the following relations among the differential operators $D_{1}, D_{2}, D_{3}, D_{4}$.

$$
\begin{array}{lll}
D_{1} D_{2}=0, & D_{1} D_{3}=0, & D_{1} D_{4}=D_{4} D_{2}-(n-2 p) D_{4}, \\
D_{2} D_{1}=0, & D_{2} D_{3}=D_{3} D_{1}+(n-2 p) D_{3}, & D_{2} D_{4}=0, \\
D_{3} D_{2}=0, & D_{3} D_{3}=0, & D_{3} D_{4}=D_{2}^{2}-(n-2 p) D_{2}, \\
D_{4} D_{1}=0, & D_{4} D_{3}=D_{1}^{2}+(n-2 p) D_{1}, & D_{4} D_{4}=0 .
\end{array}
$$

4. The Order of the Operators in $\mathcal{D}(W)$

In this section we prove that any operator

$$
D=\sum_{\ell=0}^{s} \partial^{\ell} F_{\ell}(x) \in \mathcal{D}(W)
$$


is of even order and either $F_{s}=\mathbf{0}$ or $\operatorname{deg}\left(F_{s}\right)=s$. Furthermore, $\left\{D_{1}, D_{2}, D_{3}, D_{4}\right\}$ generates the algebra $\mathcal{D}(W)$. We start by considering operators $D$ such that $\widetilde{D}=D$, later we prove that the same results hold for all operators.

From (7) and (8) we have

$$
\begin{aligned}
\left(B_{w-2(k-i)}^{w}\right)_{11} & =\frac{p+w-2 k+2 i}{p+w-2 k} \frac{(-1)^{i} 2^{2 i}\left(\frac{n+1}{2}+w-k\right)_{i}[k]_{i}}{[w-2 k+2 i]_{2 i}}\left(B_{w-2 k}^{w}\right)_{11}, \\
\left(B_{w-2 k-1}^{w}\right)_{12} & =\frac{w-2 k}{p+w-2 k}\left(B_{w-2 k}^{w}\right)_{11} .
\end{aligned}
$$

Let us assume that $\widetilde{D}=D$ and that $s=2 r+1, r \in \mathbb{N}$. If, for $0 \leq \ell \leq s$, we denote $F_{\ell}=\sum_{i=0}^{\ell} x^{i} F_{i}^{\ell}$ then from our hypotheses we have

$$
F_{\ell-2 i}^{\ell}=\left(\begin{array}{cc}
u_{\ell-2 i}^{\ell} & 0 \\
0 & v_{\ell-2 i}^{\ell}
\end{array}\right), \quad F_{\ell-2 i+1}^{\ell}=\left(\begin{array}{cc}
0 & y_{\ell-2 i+1}^{\ell} \\
z_{\ell-2 i+1}^{\ell} & 0
\end{array}\right) .
$$

If we put $m=2 k$, Proposition 3.2 gives us that

$$
\begin{array}{r}
\sum_{i=0}^{r} B_{w-2 k+2 i}^{w}\left(\sum_{\ell=0}^{s}[w-2 k+2 i]_{\ell} F_{\ell-2 i}^{\ell}\right)+\sum_{i=1}^{r+1} B_{w-2 k+2 i-1}^{w}\left(\sum_{\ell=0}^{s}[w-2 k+2 i-1]_{\ell} F_{\ell-2 i-1}^{\ell}\right) \\
-\left(\sum_{\ell=0}^{s}[w]_{\ell} F_{\ell}^{\ell}\right) B_{w-2 k}^{w}=0
\end{array}
$$

for $0 \leq 2 k \leq w$.

Then, looking at the entry $(1,1)$ and using (10) and (11) we have

$$
\begin{array}{r}
\sum_{i=0}^{r} \frac{p+w-2 k+2 i}{p+w-2 k} \frac{(-1)^{i} 2^{2 i}\left(\frac{n+1}{2}+w-k\right)_{i}[k]_{i}}{[w-2 k+2 i]_{2 i}}\left(B_{w-2 k}^{w}\right)_{11}\left(\sum_{\ell=2 i}^{s}[w-2 k+2 i]_{\ell} u_{\ell-2 i}^{\ell}\right) \\
+\sum_{i=1}^{r+1} \frac{w-2 k+2 i}{p+w-2 k} \frac{(-1)^{i} 2^{2 i}\left(\frac{n+1}{2}+w-k\right)_{i}[k]_{i}}{[w-2 k+2 i]_{2 i}}\left(B_{w-2 k}^{w}\right)_{11}\left(\sum_{\ell=2 i-1}^{s}[w-2 k+2 i-1]_{\ell} z_{\ell-2 i+1}^{\ell}\right) \\
-\sum_{\ell=0}^{s}[w]_{\ell} u_{\ell}^{\ell}\left(B_{w-2 k}^{w}\right)_{11}=0 .
\end{array}
$$

for $0 \leq 2 k \leq w$. Hence

$$
\begin{aligned}
\sum_{i=0}^{r}(p+ & w-2 k+2 i)(-1)^{i} 2^{2 i}\left(\frac{n+1}{2}+w-k\right)_{i}[k]_{i}\left(\sum_{\ell=2 i}^{s}[w-2 k]_{\ell-2 i} u_{\ell-2 i}^{\ell}\right) \\
& +\sum_{i=1}^{r+1}(-1)^{i} 2^{2 i}\left(\frac{n+1}{2}+w-k\right)_{i}[k]_{i}\left(\sum_{\ell=2 i-1}^{s}[w-2 k]_{\ell-2 i+1} z_{\ell-2 i+1}^{\ell}\right)-\sum_{\ell=0}^{s}[w]_{\ell} u_{\ell}^{\ell}(p+w-2 k)=0 .
\end{aligned}
$$

for $0 \leq 2 k \leq w$.

Let us consider $w=\widetilde{m}+2 k$, therefore for any $\widetilde{m}$ and $k$ in $\mathbb{N}_{0}$ we have

$$
\begin{aligned}
& \sum_{i=0}^{r}(-1)^{i} 2^{2 i}\left(\frac{n+1}{2}+\widetilde{m}+k\right)_{i}[k]_{i}(p+\widetilde{m}+2 i) \sum_{\ell=2 i}^{s}[\widetilde{m}]_{\ell-2 i} u_{\ell-2 i}^{\ell} \\
& \quad+\sum_{i=1}^{r+1}(-1)^{i} 2^{2 i}\left(\frac{n+1}{2}+\widetilde{m}+k\right)_{i}[k]_{i} \sum_{\ell=2 i-1}^{s}[\widetilde{m}]_{\ell-2 i+1} z_{\ell-2 i+1}^{\ell}-(p+\widetilde{m}) \sum_{\ell=0}^{s}[\widetilde{m}+2 k]_{\ell} u_{\ell}^{\ell}=0 .
\end{aligned}
$$


THE ALGEBRA OF DIFFERENTIAL OPERATORS FOR A MATRIX WEIGHT. AN ULTRASPHERICAL EXAMPLE. 9

If we consider (12) as a polynomial in $k$ then we obtain

$$
z_{0}^{s}=0=u_{s}^{s},
$$

by looking at the terms of degree $s+1$ and $s$.

We observe that (12) is a polynomial equation in two variables $\widetilde{m}$ and $k$ and its term of highest total degree gives us that the following equation holds.

$$
\sum_{i=1}^{r}(-1)^{i} 2^{2 i}(\widetilde{m}+k)^{i} k^{i} \widetilde{m}^{s-2 i+1}\left(u_{s-2 i}^{s}+z_{s-2 i+1}^{s}\right)=0
$$

thus

$$
\sum_{i=1}^{r} \sum_{j=0}^{i}\left(\begin{array}{l}
i \\
j
\end{array}\right)(-1)^{i} 2^{2 i}(\widetilde{m})^{i-j} k^{j} k^{i} \widetilde{m}^{s-2 i+1}\left(u_{s-2 i}^{s}+z_{s-2 i+1}^{s}\right)=0
$$

hence

$$
\sum_{i=1}^{r} \sum_{j=0}^{i}\left(\begin{array}{l}
i \\
j
\end{array}\right)(-1)^{i} 2^{2 i} k^{j+i} \widetilde{m}^{s-i-j+1}\left(u_{s-2 i}^{s}+z_{s-2 i+1}^{s}\right)=0 .
$$

Then, for any $1 \leq q \leq r$ we observe that the term of degree $q$ in the variable $k$ is

$$
\sum_{\frac{q}{2} \leq i \leq q}\left(\begin{array}{c}
i \\
q-i
\end{array}\right)(-1)^{i} 2^{2 i} k^{q} \tilde{m}^{s-q+1}\left(u_{s-2 i}^{s}+z_{s-2 i+1}^{s}\right)=0 .
$$

Hence, for any $1 \leq q \leq r$ we have

$$
\sum_{\frac{q}{2} \leq i \leq q}\left(\begin{array}{c}
i \\
q-i
\end{array}\right)(-1)^{i} 2^{2 i}\left(u_{s-2 i}^{s}+z_{s-2 i+1}^{s}\right)=0 .
$$

By letting $q=1$ in (15) we have $u_{s-2}^{s}+z_{s-1}^{s}=0$. Then, by letting $q=2$ also in (15) we have $u_{s-4}^{s}+z_{s-3}^{s}=0$. Inductively we have

$$
u_{s-2 i}^{s}=-z_{s-2 i+1}^{s}, \text { for } 1 \leq i \leq r .
$$

In a completely analogous way one finds that

$$
y_{0}^{s}=0=v_{s}^{s}
$$

and that

$$
v_{s-2 i}^{s}=-y_{s-2 i+1}^{s}, \text { for } 1 \leq i \leq r .
$$

On the other hand, from Proposition 3.5, we know that $\Lambda_{w}(D)$ is a diagonal matrix for any $w$, thus $D$ commutes with $D_{1}$. By looking at the highest order derivative in the equation $D D_{1}=D_{1} D$ we obtain

$$
\left(\begin{array}{cc}
x^{2} & x \\
-x & -1
\end{array}\right) F_{s}=F_{s}\left(\begin{array}{cc}
x^{2} & x \\
-x & -1
\end{array}\right)
$$

i.e.

$$
\left(\begin{array}{cc}
x^{2} & x \\
-x & -1
\end{array}\right) \sum_{k=0}^{s} x^{k} F_{k}^{s}=\sum_{k=0}^{s} x^{k} F_{k}^{s}\left(\begin{array}{cc}
x^{2} & x \\
-x & -1
\end{array}\right),
$$

then, for any $0 \leq k \leq s$, we have

$$
\left(\begin{array}{cc}
0 & 0 \\
0 & -1
\end{array}\right) F_{k}^{s}+\left(\begin{array}{cc}
0 & 1 \\
-1 & 0
\end{array}\right) F_{k-1}^{s}+\left(\begin{array}{ll}
1 & 0 \\
0 & 0
\end{array}\right) F_{k-2}^{s}=F_{k}^{s}\left(\begin{array}{cc}
0 & 0 \\
0 & -1
\end{array}\right)+F_{k-1}^{s}\left(\begin{array}{cc}
0 & 1 \\
-1 & 0
\end{array}\right)+F_{k-2}^{s}\left(\begin{array}{ll}
1 & 0 \\
0 & 0
\end{array}\right) .
$$


If $k$ and $s$ are of the same parity, say $k=s-2 i+2$, then (19) becomes

$$
\left(\begin{array}{cc}
0 & 1 \\
-1 & 0
\end{array}\right) F_{s-2 i+1}^{s}=F_{s-2 i+1}^{s}\left(\begin{array}{cc}
0 & 1 \\
-1 & 0
\end{array}\right)
$$

whence, looking at the entry $(1,1)$, we obtain

$$
z_{s-2 i+1}^{s}=-y_{s-2 i+1}^{s}, \text { for } 1 \leq i \leq r+1 .
$$

If $k$ and $s$ are of different parity, say $k=s-2 i+1$, then the entry $(1,2)$ of the matrix equation (19) gives us

$$
-z_{s-2 i+1}+u_{s-2 i}=-v_{s-2 i}+z_{s-2 i-1},
$$

which combined with (16), (18) and (20) becomes

$$
u_{s-2 i}^{s}=-u_{s-2 i-2}^{s}
$$

Furthermore, using (16), (18) and (20) it is clear that the values $u_{1}^{s}, u_{3}^{s}, \ldots, u_{s}^{s}$ determine $F_{s}$. Using (21) it is straightforward to see that the value of $u_{s}^{s}$ determines all the other entries of $F_{s}$ and that, since $u_{s}^{s}=0$, we have $F_{s}=\mathbf{0}$. Therefore, there is no operator $D$ of odd order in $\mathcal{D}(W)$ such that $\widetilde{D}=D$.

Even more, since $F_{s}$ is zero (12) turns out to be

$$
\begin{aligned}
& \sum_{i=0}^{r}(-1)^{i} 2^{2 i}\left(\frac{n+1}{2}+\widetilde{m}+k\right)_{i}[k]_{i}(p+\widetilde{m}+2 i) \sum_{\ell=2 i}^{s-1}[\widetilde{m}]_{\ell-2 i} u_{\ell-2 i}^{\ell} \\
& \quad+\sum_{i=1}^{r}(-1)^{i} 2^{2 i}\left(\frac{n+1}{2}+\widetilde{m}+k\right)_{i}[k]_{i} \sum_{\ell=2 i-1}^{s-1}[\widetilde{m}]_{\ell-2 i+1} z_{\ell-2 i+1}^{\ell}-(p+\widetilde{m}) \sum_{\ell=0}^{s-1}[\widetilde{m}+2 k]_{\ell} u_{\ell}^{\ell}=0
\end{aligned}
$$

If we denote $s^{\prime}=s-1$ and observe the term of highest total degree in the variables $\widetilde{m}$ and $k$, we obtain the following equation,

$$
\sum_{i=1}^{r}(-1)^{i} 2^{2 i}(\widetilde{m}+k)^{i} k^{i} \widetilde{m}^{s^{\prime}-2 i+1}\left(u_{s^{\prime}-2 i}^{s^{\prime}}+z_{s^{\prime}-2 i+1}^{s^{\prime}}\right)-\widetilde{m}(\widetilde{m}+2 k)^{s^{\prime}} u_{\ell}^{\ell}=0 .
$$

If $u_{s^{\prime}}^{s^{\prime}}=0$ then this equation is exactly equation (14) with $s^{\prime}$ in place of $s$; therefore with the same procedure as before we obtain an equation similar to (16), with $s^{\prime}$ in place of $s$; if $v_{s^{\prime}}^{s^{\prime}}=0$, then we have also an equation similar to (18). Equation (19) and the subsequent equations (20) and (21) are obtained independently of the parity of $s$, therefore they remain valid for $s^{\prime}$, having then that if $u_{s^{\prime}}^{s^{\prime}}=v_{s^{\prime}}^{s^{\prime}}=0$ then $F_{s^{\prime}}=\mathbf{0}$.

Finally, for a given operator $D=\sum_{\ell=0}^{s} \partial^{\ell} F_{\ell}(x) \in \mathcal{D}(W)$, with $\widetilde{D}=D$, of even order $s=2 r$ we can consider the operator

$$
D-u_{s}^{s} D_{1}^{r}-v_{s}^{s} D_{2}^{r}
$$

which has to be of order $s-2$. Inductively it can be easily seen that the set of operators

$$
\left\{D_{1}, D_{2}\right\}
$$

generates the subalgebra of all the operators in $\mathcal{D}(W)$ such that $\widetilde{D}=D$.

The following theorem summarizes all the results obtained up to this point in this section.

Theorem 4.1. Any operator $D=\sum_{\ell=0}^{s} \partial^{\ell} F_{\ell} \in \mathcal{D}(W)$ of order $s$, such that $\widetilde{D}=D$, is of even order and the polynomial $F_{s}$ is of degree $s$.

Furthermore, the operators $D_{1}$ and $D_{2}$ generate the subalgebra $\{D \in \mathcal{D}(W): \widetilde{D}=D\}$ of $\mathcal{D}(W)$.

Corollary 4.2. Any operator $D=\sum_{\ell=0}^{s} \partial^{\ell} F_{\ell} \in \mathcal{D}(W)$ of order $s$, such that $\widetilde{D}=-D$, is of even order and the polynomial $F_{s}$ is of degree $s$. 
THE ALGEBRA OF DIFFERENTIAL OPERATORS FOR A MATRIX WEIGHT.

Proof. Given an operator $D=\sum_{\ell=0}^{s} \partial^{\ell} F_{\ell} \in \mathcal{D}(W)$ of order $s$ such that $\widetilde{D}=-D$ let us consider the operator

$$
D_{0}=D\left(D_{3}+D_{4}\right) \text {. }
$$

Since $\widetilde{D}_{3}=-D_{3}$ and $\widetilde{D}_{4}=-D_{4}$ we have that $\widetilde{D}_{0}=D_{0}$. Also, the highest order term of $D_{3}+D_{4}$ is $\partial^{2}\left(x^{2}-1\right)\left(\begin{array}{ll}0 & 1 \\ 1 & 0\end{array}\right)$, therefore the order of $D_{0}$ is $s+2$, which implies, by Theorem 4.1 , that $s+2$ is even and that if $D_{0}=\sum_{\ell=0}^{s+2} \partial^{\ell} G_{\ell}$ then $G_{s+2}=\left(1-x^{2}\right) F_{s}$ is a polynomial of degree $s+2$, hence $F_{s}$ is a polynomial of order $s$.

Now we give some results that are pretty technical. In the proofs we strongly use the relations in Remark 3.8. In particular we have

$$
\left(D_{1}+D_{2}\right)^{k} D_{1}=D_{1}^{k+1}, \quad\left(D_{1}+D_{2}\right)^{k} D_{2}=D_{2}^{k+1}, \quad\left(D_{1}+D_{2}\right)^{k} D_{3}=D_{2}^{k} D_{3}, \quad\left(D_{1}+D_{2}\right)^{k} D_{4}=D_{1}^{k} D_{4},
$$

thus, looking at (9), it is also clear that

$$
\left\{\left(D_{1}+D_{2}\right)^{i} D_{1},\left(D_{1}+D_{2}\right)^{i} D_{2},\left(D_{1}+D_{2}\right)^{i} D_{3},\left(D_{1}+D_{2}\right)^{i} D_{4}, \mathbf{I}: i \in \mathbb{N}_{0}\right\}
$$

is a linear independent set over $\mathbb{C}$.

Theorem 4.3. The algebra $\mathcal{D}(W)$ is generated by $\left\{D_{1}, D_{2}, D_{3}, D_{4}\right\}$. If $D=\sum_{\ell=0}^{s} \partial^{\ell} F_{\ell} \in \mathcal{D}(W)$ with $F_{s} \neq \mathbf{0}$ then $s$ is even and $F_{s}$ is of degree $s$.

Proof. For any $D \in \mathcal{D}(W)$ we have that $D=E_{1}+E_{2}$, with $\widetilde{E}_{1}=E_{1}$ and $\widetilde{E}_{2}=-E_{2}$. Thus, it only remains to prove that if $D=\sum_{\ell=0}^{s} \partial^{\ell} F_{\ell} \in \mathcal{D}(W)$, with $\widetilde{D}=-D$, then it is generated by $\left\{D_{1}, D_{2}, D_{3}, D_{4}\right\}$. Let $F_{s}=\sum_{j=0}^{s} F_{j}^{s}$. From Corollary 4.2 we know that $s$ is even and from the relation $\widetilde{D}=-D$ it follows that $\left(F_{s}^{s}\right)_{11}=\left(F_{s}^{s}\right)_{22}=0$. If $s \leq 2$ the assertion is already proved by Lemma 3.7. if $s \geq 4$ we consider the operator

$$
D_{0}=D-\left(D_{1}+D_{2}\right)^{\frac{s}{2}-1}\left(\left(F_{s}^{s}\right)_{21} D_{3}+\left(F_{s}^{s}\right)_{12} D_{4}\right) \text {. }
$$

It can be easily checked that $\widetilde{D}_{0}=-D_{0}$ and that $D_{0}=\sum_{\ell=0}^{s} \partial^{\ell} G_{\ell}$ with $G_{s}$ is of degree less than $s$ and therefore, by Corollary $4.2, G_{s}=0$, then $D_{0}$ is an operator of order at most $s-2$, hence by induction we obtain the statement in the theorem.

With a very similar proof we obtain the following corollary.

Corollary 4.4. The vector space $\mathcal{D}(W)$ is spanned by the set

$$
\left\{\left(D_{1}+D_{2}\right)^{i} D_{1},\left(D_{1}+D_{2}\right)^{i} D_{2},\left(D_{1}+D_{2}\right)^{i} D_{3},\left(D_{1}+D_{2}\right)^{i} D_{4}, \mathbf{I}: i \in \mathbb{N}_{0}\right\} .
$$

Proof. Let $D \in \mathcal{D}(W)$, then it is of the form

$$
D=\sum_{\ell=0}^{2 r} \partial^{\ell} F_{\ell},
$$

for some $r \in \mathbb{N}_{0}$, with $F_{s}=\sum_{j=0}^{s} F_{j}^{s}$. Let us consider the operator

$$
D_{0}=D-\left(D_{1}+D_{2}\right)^{k-1}\left(\left(F_{s}^{s}\right)_{11} D_{1}+\left(F_{s}^{s}\right)_{22} D_{2}+\left(F_{s}^{s}\right)_{21} D_{3}+\left(F_{s}^{s}\right)_{12} D_{4}\right) .
$$

It can be easily checked that if $D_{0}=\sum_{\ell=0}^{s} \partial^{\ell} G_{\ell}$ then $G_{s}$ is of degree smaller than $s$, and therefore by Theorem $4.3 D_{0}$ is an operator of order at most $s-2$. Again, by induction we obtain the statement in the theorem.

Corollary 4.5. $\mathcal{D}(W)$ is a free left-module over the subalgebra generated by $D_{1}+D_{2}$. The following set is a basis

$$
\left\{\mathbf{I}, D_{2}, D_{3}, D_{4}\right\} \text {. }
$$

As a direct consequence of Proposition 2.2 and Theorem 4.3 we have

Corollary 4.6. For any $D \in D(W)$, the order of $D$ equals the degree of $\Lambda_{w}(D)$ as a polynomial in $w$. 


\section{Structure of $\mathcal{D}(W)$}

In this section we go deeper into the structure of the algebra $\mathcal{D}(W)$. Also, we prove that the non-similar weights $W_{p, n}$ have isomorphic algebras $\mathcal{D}\left(W_{p, n}\right)$.

Theorem 5.1. The algebra $\mathcal{D}(W)$ is generated by

$$
\left\{D_{1}+D_{2}, D_{3}+D_{4}\right\}
$$

Proof. From Remark 3.8 we have that

$$
\left(D_{3}+D_{4}\right)^{2}=\left(D_{1}+D_{2}\right)^{2}+(n-2 p)\left(D_{1}-D_{2}\right),
$$

therefore $\left\{D_{1}+D_{2}, D_{3}+D_{4}\right\}$ generates $D_{1}$ and $D_{2}$.

From

$$
\left(D_{3}+D_{4}\right) D_{1}=D_{3} D_{1}=D_{2} D_{3}-(n-2 p) D_{3}=D_{2}\left(D_{3}+D_{4}\right)-(n-2 p) D_{3}
$$

we have that $\left\{D_{1}+D_{2}, D_{3}+D_{4}\right\}$ generates also $D_{3}$ and $D_{4}$. The theorem follows from Theorem 4.3 ,

Let us define

$$
A=\left(D_{1}+D_{2}\right) /(2 n-p), \quad B=\left(D_{3}+D_{4}\right) /(2 n-p) .
$$

Therefore we have the following expressions for the operators $D_{1}, D_{2}, D_{3}, D_{4}$ (see Remark 3.8)

$$
\begin{array}{ll}
D_{1}=\left(B^{2}-A^{2}+A\right)(n-2 p) / 2, & D_{2}=\left(A^{2}-B^{2}+A\right)(n-2 p) / 2, \\
D_{3}=(-B A+A B+B)(n-2 p) / 2, & D_{4}=(B A-A B+B)(n-2 p) / 2 .
\end{array}
$$

Let us call $\mathcal{A}$ to the subalgebra generated by $A$.

Lemma 5.2. The set $S=\left\{\mathbf{I}, B^{2}, B, B A\right\}$ is also a basis of $\mathcal{D}(W)$ as a left-module over $\mathcal{A}$.

Proof. It is clear from (22) and Corollary 4.5 that $S$ generates $\mathcal{D}(W)$. Let assume that

$$
m_{1} I+m_{2} B^{2}+m_{3} B+m_{4} B A=0
$$

for some $m_{1}, m_{2}, m_{3}, m_{4} \in \mathcal{A}$. From (22) we observe that

$$
B^{2}=A^{2}+A-2(n-2 p)^{-1} D_{2}, \quad B A=\left(D_{4}-D_{3}\right)(n-2 p)^{-1}+A\left(D_{3}+D_{4}\right),
$$

then we have

$$
\begin{array}{r}
\left(m_{1}+m_{2} A^{2} I+A\right) \mathbf{I}+m_{2}\left(-2(n-2 p)^{-1}\right) D_{2}+\left(m_{3}+m_{4}\left(-(n-2 p)^{-1} \mathbf{I}+A\right)\right) D_{3}+ \\
\left(m_{3}+m_{4}\left((n-2 p)^{-1} \mathbf{I}+A\right)\right) D_{4}=0,
\end{array}
$$

whence it follows in a straightforward way that $m_{1}=m_{2}=m_{3}=m_{4}=0$.

It is worth to observe that the following relations hold:

$$
\begin{aligned}
B^{2} A-A B^{2} & =0, \\
B A^{2}+A^{2} B-2 A B A-B & =0, \\
B A B+A^{3}-2 A B^{2}-A & =0, \\
B^{3}-2 A^{2} B+A B A & =0 .
\end{aligned}
$$

We shall next prove that the algebra $\mathcal{D}(W)$ can be described as the complex algebra generated by $A$ and $B$ subject to the relations given in (23). Let $V$ be a two dimensional complex vector space and let $T(V)$ be the corresponding tensor algebra. The identity of $T(V)$ will be also denoted by $I$. We choose a basis $\{\alpha, \beta\}$ 
of $V$ and define $I(V)$ to be the two sided ideal of $T(V)$ generated by the elements on the left-hand side of the following four equations, obtained by replacing $A$ by $\alpha$ and $B$ by $\beta$ in (23):

$$
\begin{aligned}
\beta^{2} \alpha-\alpha \beta^{2} & =0, \\
\beta \alpha^{2}+\alpha^{2} \beta-2 \alpha \beta \alpha-\beta & =0, \\
\beta \alpha \beta+\alpha^{3}-2 \alpha \beta^{2}-\alpha & =0, \\
\beta^{3}-2 \alpha^{2} \beta+\alpha \beta \alpha & =0 .
\end{aligned}
$$

Theorem 5.3. The algebra $\mathcal{D}(W)$ is isomorphic to the quotient algebra $T(V) / I(V)$.

Proof. For simplicity we will denote with the same symbol an element $T(V)$ and its canonical projection on the quotient algebra $T(V) / I(V)$. The homomorphism $\xi$ from the quotient algebra $T(V) / I(V)$ into $\mathcal{D}(W)$ defined by $\xi(\alpha)=A$ and $\xi(\beta)=B$ is surjective. Let us denote by $\mathcal{A}^{\prime}$ the subalgebra of $T(V) / I(P)$ generated by $\alpha$. It is clear that $\mathcal{A}^{\prime}$ is isomorphic to $\mathcal{A}$. The point now is to prove that $\xi$ is injective. Let $S$ denote the left-module over $\mathcal{A}$ generated by

$$
\left\{\mathbf{I}, \beta^{2}, \beta, \beta \alpha\right\} .
$$

Since $\xi$ transforms $S$ into the basis $\left\{\mathbf{I}, B^{2}, B, B A\right\}$ it is enough to prove that $S=T(V) / I(V)$. First notice that since $\alpha$ and $\beta$ satisfy (24) we have that $S$ is invariant under right multiplication by $\alpha$ and left multiplication by $\beta$. If we prove that $S$ is also left invariant under multiplication by $\beta$ we obtain the statement of the theorem.

We will prove by induction on $k$ that $\beta \alpha^{k}(\alpha \beta)$ belongs to $S$ for any $k \in \mathbb{N}_{0}$. For $k=0$ we have $\beta(\beta \alpha)=\beta^{2} \alpha=\alpha \beta^{2}$ (first equation in (24)). For $k=1$ we have $\beta \alpha(\beta \alpha)$, but we can write $\beta \alpha \beta$ as a linear combination of $\beta^{2}$ and $\mathbf{I}$ (third equation in (24)) which are in the center of the algebra, and then we can write $\beta \alpha \beta \alpha$ also as a linear combination of $\beta^{2}$ and $\mathbf{I}$. Assume that $\beta \alpha^{k}(\beta \alpha)$ can be written as a linear combination of $\mathbf{I}$ and $\beta^{2}$ for $k \geq 1$ and let us consider the case $k+1$; since $\beta \alpha \alpha$ can be written as $p(\alpha) \beta+q(\alpha) \beta \alpha$ with $p, q$ polynomials (last equation in (24)), we have

$$
\beta \alpha^{k+1} \beta \alpha=(p(\alpha) \beta+q(\alpha) \beta \alpha) \alpha^{k-1} \beta \alpha=p(\alpha) \beta \alpha^{k-1} \beta \alpha+q(\alpha) \beta \alpha^{k} \beta \alpha,
$$

which, by the inductive hypothesis, is a linear combination of $\mathbf{I}$ and $\beta^{2}$. Then $\beta \alpha^{k}(\alpha \beta)$ belongs to $S$ for any $k \in \mathbb{N}_{0}$.

In a very similar way it can be proved that $\beta \alpha^{k}(\beta), \beta \alpha^{k}\left(\beta^{2}\right)$ and $\beta \alpha^{k}(\mathbf{I})$ belongs to $S$ for any $k \in \mathbb{N}_{0}$. Therefore, $S$ is stable under left multiplication by $\beta$ and the theorem is proved.

Notice that if one has two similar weights $W$ and $\widetilde{W}$, then the corresponding differential operator algebras are isomorphic: Let $M$ be an invertible matrix such that $\widetilde{W}=M W M^{*}$, it can be easily checked that $D \rightarrow M D M^{-1}$ is an isomorphism from $\mathcal{D}(W)$ onto $\mathcal{D}(\widetilde{W})$. The converse is not true. Lemma 3.1 states that for $(p, n) \neq\left(p^{\prime}, n^{\prime}\right)$ the weights $W_{p, n}$ and $W_{p^{\prime}, n^{\prime}}$ are non-similar, but relations (24) do not depend on the parameters $n$ and $p$. Thus, we have the following result.

Theorem 5.4. For any two matrix weights $W=W_{p, n}$ and $W^{\prime}=W_{p^{\prime}, n^{\prime}}$ the algebras $\mathcal{D}(W)$ and $\mathcal{D}\left(W^{\prime}\right)$ are isomorphic.

Theorem 5.5. The algebra $\mathcal{D}(W)$ is isomorphic to the algebra $\mathcal{D}(\tilde{W})$ studied in Tir11, with $\tilde{W}=e^{-t^{2}} e^{t M} e^{t M^{*}}$ where

$$
M=\left(\begin{array}{ll}
0 & a \\
0 & 0
\end{array}\right), \quad a \in \mathbb{C} \backslash\{0\},
$$

is isomorphic to the algebra $\mathcal{D}(W)$ considered in the present paper. 
Proof. In Tir11] it is proved that the algebra $\mathcal{D}(\tilde{W})$ is the complex algebra generated by two elements $E, F$ subject to certain relations given by equations (109)-(114) (page 321 in [Tir11]). We observe that equations (110) and (112) in [Tir11] have minor typos, they actually should be:

$$
\begin{array}{r}
F^{4} / 16-a^{2} F^{2} E^{2} / 2-2 a F^{2} E-2 F^{2}+a^{4} E^{4}+8 a^{3} E^{3}-a^{2}\left(a^{2}-24\right) E^{2}-4 a\left(a^{2}-8\right) E-4\left(a^{2}-4\right) \mathbf{I}=0, \\
F^{3}+F^{2}[E, F]-4 a^{2} E^{2} F-4 a^{2} E^{2}[E, F]+4 a(a-4) E F+ \\
4 a(a-4) E[E, F]+8(a-2) F+8(a-2)[E, F]=0,
\end{array}
$$

respectively.

Let us consider the algebra morphism $\varphi: \mathcal{D}(W) \rightarrow \mathcal{D}(\tilde{W})$ defined by

$$
\varphi(A)=E+2 \mathbf{I} /|a|^{2}, \quad \varphi(B)=\left(F\left(4+|a|^{4}\right)+[E, F]\left(4-|a|^{4}\right)\right) /\left(8|a|^{4}\right),
$$

and the algebra morphism $\xi: \mathcal{D}(W) \rightarrow \mathcal{D}(\tilde{W})$ defined by

$$
\xi(E)=A-2 \mathbf{I} /|a|^{2}, \quad \xi(F)=\left(4+|a|^{4}\right) B+\left(4-|a|^{4}\right)[B, A],
$$

Using (23) and (109)-(114) (from [Tir11]), it is straightforward to see that $\varphi$ and $\xi$ are well defined and that $\varphi$ is an isomorphism with inverse $\xi$.

\section{The center of $\mathcal{D}(W)$}

In this section we determine the center of our algebra and we will analyze the structure of $\mathcal{D}(W)$ as a module over its center.

Let us define

$$
C_{1}=\left(D_{3}+D_{4}\right)^{2}, \quad C_{2}=D_{3} D_{1} D_{4}+D_{4}\left(D_{2}-(n-2 p) \mathbf{I}\right) D_{3} .
$$

From Lemma 3.7 it follows that

$$
\begin{aligned}
& \Lambda_{w}\left(C_{1}\right)=p_{1}(w) \mathbf{I}=(w+p)(w+p+1)(w+n-p+1)(w+n-p) \mathbf{I}, \\
& \Lambda_{w}\left(C_{2}\right)=p_{2}(w) \mathbf{I}=(w+p)(w+p+1)^{2}(w+n-p+1)(w+n-p)^{2} \mathbf{I},
\end{aligned}
$$

whence it is clear that $C_{1}$ and $C_{2}$ are in the center of $\mathcal{D}(W)$. Also, we observe that they are not algebraically independent since the following relations hold:

$$
C_{1}^{3}-C_{2}^{2}=(n-2 p) C_{1} C_{2} .
$$

Theorem 6.1. The center $\mathcal{Z}(W)$ of $\mathcal{D}(W)$ is generated by $C_{1}$ and $C_{2}$ and is isomorphic to the affine algebra of the following singular algebraic curve:

$$
x^{3}-y^{2}=(n-2 p) x y .
$$

Proof. Let us assume that $X$ is an element in $\mathcal{Z}(W) \subset \mathcal{D}(W)$, let $s$ be the order of $X$. Since $\Lambda_{w}(X) \Lambda_{w}\left(D_{1}\right)=$ $\Lambda_{w}\left(D_{1}\right) \Lambda_{w}(X)$ we have that $\Lambda_{w}(X)$ is a diagonal matrix for every $w$ (see Theorem 3.7 for the expression of $\left.\Lambda_{w}\left(D_{1}\right)\right)$. From $\Lambda_{w}(X) \Lambda_{w}\left(D_{3}\right)=\Lambda_{w}\left(D_{3}\right) \Lambda_{w}(X)$ we obtain that $\Lambda_{w}(X)$ is a scalar matrix, i.e.

$$
\Lambda_{w}(X)=p_{X}(w) \mathbf{I}
$$

with $p_{X}(w)$ a polynomial on $w$. From Corollary 4.6 we have that the degree of $p_{X}$ is equal to the order of $X$, which has to be an even number $s$ (Theorem 4.3). If $s=0$ there is nothing to prove; it is clear that $s \neq 2$ since all the operators of order 2 in $\mathcal{D}(W)$ are a linear combination of $\left\{D_{1}, D_{2}, D_{3}, D_{4}\right.$, I $\}$ (see Theorem 3.7). We will complete the proof by induction on the even number $s$ : assume that $s \geq 4$, since the polynomials $p_{1}$ and $p_{2}$ are of degree 4 and 6 respectively we can find integers $s_{1} \in \mathbb{N}_{0}$ and $s_{2} \in\{0,1\}$ such that the polynomial $p_{\tilde{X}}=p_{X}-p_{1}^{s_{1}} p_{2}^{s_{2}}$ is of degree smaller than $s$. Therefore, by the inductive hypothesis, the operator $\tilde{X}=X-C_{1}{ }^{s_{1}} C_{2}{ }^{s_{2}}$ is a polynomial in $C_{1}$ and $C_{2}$ and hence also the operator $X$. 
THE ALGEBRA OF DIFFERENTIAL OPERATORS FOR A MATRIX WEIGHT.

Corollary 6.2. For every operator $D \in \mathcal{Z}(W)$ there exists a unique pair of polynomials $p$ and $q$ such that

$$
D=p\left(C_{1}\right)+q\left(C_{1}\right) C_{2} .
$$

Theorem 6.3. The algebra $\mathcal{D}(W)$ is a finitely generated torsion-free module over the ring $\mathcal{Z}(W)$. It is not flat and hence is not projective.

Proof. Torsion free is clear since for every operator $D$ in $\mathcal{Z}(W)$ the eigenvalue $\Lambda_{w}(D)$ is a scalar matrix. From Corollary 4.4, since $C_{1}=\left(D_{3}+D_{4}\right)^{2}=\left(D_{1}+D_{2}\right)^{2}+(n-2 p)\left(D_{1}-D_{2}\right)$ (see Remark 3.8), it is clear that $\mathcal{D}(W)$ is finitely generated over the subalgebra generated by $C_{1}$ and therefore by $\mathcal{Z}(W)$. Given that the center $\mathcal{Z}(W)$ is a Noetherian ring, $\mathcal{D}(W)$ is projective (resp. flat) if and only if it is locally free.

We will prove that $\mathcal{D}(W)$ is not locally free. Let $\mathfrak{m}$ the maximal ideal generated by $C_{1}$ and $C_{2}$, then the complement $S$ are the operators of the form $p\left(C_{1}\right)+q\left(C_{1}\right) C_{2}$ with $p$ and $q$ polynomials such that the constant term of $p$ is nonzero; i.e. $S$ is the set of operators $D$ in $\mathcal{Z}(W)$ such that the polynomial $\Lambda_{w}\left(C_{1}\right)$ does not divides $\Lambda_{w}(D)$ (recall from (25) that $\Lambda_{w}\left(C_{1}\right)$ divides $\Lambda_{w}\left(C_{2}\right)$ ).

Let us assume that $\left\{A_{i}\right\}_{j \in J}$ is a basis of the localization of $\mathcal{D}(W)$ with respect to $\mathfrak{m}$, called $\mathcal{D}(W)_{\mathfrak{m}}$, over the ring $\mathcal{Z}(W)_{\mathfrak{m}}$, the localization of $\mathcal{Z}(W)$ by $\mathfrak{m}$. Therefore,

$$
D_{3}=\sum_{j \in J} C_{1, j} A_{j}, \quad D_{2} D_{3}=\sum_{j \in J} C_{2, j} A_{j},
$$

with $C_{i, j}$ of the form

$$
C_{i, j}=\frac{p_{i, j}\left(C_{1}\right)+q_{i, j}\left(C_{1}\right) C_{2}}{p_{i, j}^{\prime}\left(C_{1}\right)+q_{i, j}^{\prime}\left(C_{1}\right) C_{2}}
$$

with $p_{i, j}, q_{i, j}, p_{i, j}^{\prime}, q_{i, j}^{\prime}$ polynomials for all $i=1,2$ and $j \in J$, such that the constant coefficient of $p_{i, j}^{\prime}$ is nonzero.

Notice that there exists $j_{0}$ such that $p_{2, j_{0}}$ has a nonzero constant term, in other words $p_{j_{0}} \in S$. By considering the eigenvalues in (26) we have

$$
\begin{aligned}
& \Lambda_{w}\left(D_{2}\right) \Lambda_{w}\left(D_{3}\right) \prod_{j \in J}\left(p_{2, j}^{\prime}\left(\Lambda_{w}\left(C_{1}\right)\right)+q_{2, j}^{\prime}\left(\Lambda_{w}\left(C_{1}\right)\right) \Lambda_{w}\left(C_{2}\right)\right) \\
& \left.\quad=\sum_{j \in J}\left(\prod_{k \neq j}\left(p_{2, k}^{\prime}\left(\Lambda_{w}\left(C_{1}\right)\right)+q_{2, k}^{\prime}\left(\Lambda_{w}\left(C_{1}\right)\right) \Lambda_{w}\left(C_{2}\right)\right)\right)\left(p_{2, j}\left(\Lambda_{w}\left(C_{1}\right)\right)+q_{2, j}\left(\Lambda_{w}\left(C_{1}\right)\right) \Lambda_{w}\left(C_{2}\right)\right)\right) \Lambda_{w}\left(A_{j}\right),
\end{aligned}
$$

if $p_{2, j}$ had a zero constant term for every $j$ then we would have that the polynomial $w+n-p+1$ divides the right-hand side of the equation above since it divides $\Lambda_{w}\left(C_{1}\right)$ and $\Lambda_{w}\left(C_{2}\right)$, hence we would have that it also divides the left-hand side, which is clearly false (see (9).

Given that

$$
C_{1} D_{2} D_{3}-C_{2} D_{3}=0
$$

we have

$$
C_{1} \sum_{j \in J} C_{2, j} A_{j}-C_{2} \sum_{j \in J} C_{1, j} A_{j}=0
$$

in particular

$$
C_{1} C_{2, j_{0}}-C_{2} C_{1, j_{0}}=0 .
$$

Now, we only work out the last identity. Denoting $p_{i, j}, q_{i, j}, p_{i, j}^{\prime}, q_{i, j}^{\prime}$ the respective polynomials given by $p_{i, j}\left(C_{1}\right), q_{i, j}\left(C_{1}\right), p_{i, j}^{\prime}\left(C_{1}\right), q_{i, j}^{\prime}\left(C_{1}\right)$ (in order to simplify the notation in this part of the proof) and using that 
$C_{2}^{2}=C_{1}^{3}+(2 n-p) C_{1} C_{2}$ we have

$$
\begin{aligned}
& C_{1} C_{2, j_{0}}-C_{2} C_{1, j_{0}}=0, \\
& C_{1}\left(p_{2, j_{0}}+q_{2, j_{0}} C_{2}\right) /\left(p_{2, j_{0}}^{\prime}+q_{2, j_{0}}^{\prime} C_{2}\right)=C_{2}\left(p_{1, j_{0}}+q_{1, j_{0}} C_{2}\right) /\left(p_{1, j_{0}}^{\prime}+q_{1, j_{0}}^{\prime} C_{2}\right), \\
& C_{1}\left(p_{2, j_{0}}+q_{2, j_{0}} C_{2}\right)\left(p_{1, j_{0}}^{\prime}+q_{1, j_{0}}^{\prime} C_{2}\right)=\left(p_{1, j_{0}} C_{2}+q_{1, j_{0}} C_{2}^{2}\right)\left(p_{2, j_{0}}^{\prime}+q_{2, j_{0}}^{\prime} C_{2}\right), \\
& C_{1}\left(p_{2, j_{0}}+q_{2, j_{0}} C_{2}\right)\left(p_{1, j_{0}}^{\prime}+q_{1, j_{0}}^{\prime} C_{2}\right)=\left(p_{1, j_{0}} C_{2}+q_{1, j_{0}}\left(C_{1}^{3}-(n-2 p) C_{1} C_{2}\right)\right)\left(p_{2, j_{0}}^{\prime}+q_{2, j_{0}}^{\prime} C_{2}\right), \\
& C_{1}\left(p_{2, j_{0}}+q_{2, j_{0}} C_{2}\right)\left(p_{1, j_{0}}^{\prime}+q_{1, j_{0}}^{\prime} C_{2}\right)=\left(p_{1, j_{0}} C_{1}^{3}+\left(q_{1, j_{0}}-p_{1, j_{0}}(n-2 p) C_{1}\right) C_{2}\right)\left(p_{2, j_{0}}^{\prime}+q_{2, j_{0}}^{\prime} C_{2}\right), \\
& p_{1, j_{0}}^{\prime} C_{1} p_{2, j_{0}}+q_{1, j_{0}}^{\prime} C_{1} q_{2, j_{0}} C_{1}^{3}+C_{1}\left(p_{1, j_{0}}^{\prime} q_{2, j_{0}}+q_{1, j_{0}}^{\prime} p_{2, j_{0}}+(n-2 p) q_{1, j_{0}}^{\prime} q_{2, j_{0}}\right) C_{2}= \\
& p_{1, j_{0}} C_{1}^{3} p_{2, j_{0}}^{\prime}+\left(q_{1, j_{0}}-p_{1, j_{0}}(n-2 p) C_{1}\right) q_{2, j_{0}}^{\prime} C_{1}^{3}+ \\
&\left(p_{1, j_{0}} C_{1}^{3} q_{2, j_{0}}^{\prime}+\left(q_{1, j_{0}}-p_{1, j_{0}}(n-2 p) C_{1}\right) p_{2, j_{0}}^{\prime}+\left(q_{1, j_{0}}-p_{1, j_{0}}(n-2 p) C_{1}\right) q_{2, j_{0}}^{\prime}(n-2 p) C_{1}\right) C_{2},
\end{aligned}
$$

but the last equation contradicts Corollary 4.6 let us focus on the term without $C_{2}$, on the left-hand side of the last equation we have a polynomial on $C_{1}$ with nonzero term of degree one but on the right-hand side we have a polynomial with null term of order one.

This contradiction is a consequence of the assumption of the existence of a basis. Therefore, $\mathcal{D}(W)_{\mathfrak{m}}$ is not free. Hence $\mathcal{D}(W)$ is not locally free over $\mathcal{Z}(W)$ as asserted.

\section{ON The WeYl Algebra}

In this final section we exhibit a different way to regard the algebra $\mathcal{D}(W)$. We show that under certain circumstances, one has that the algebra $\mathcal{D}(W)$, for a given weight $W$, is the centralizer of its center in a bigger algebra, the Weyl algebra.

Notice that, for any weight $W$ of size $N$, the algebra $\mathcal{D}(W)$ is a subalgebra of the Weyl algebra $D$ over $M_{N}$ of all linear right-hand side ordinary differential operators with coefficients in $M_{N}[x]$ :

$$
\mathbf{D}=\left\{D=\sum_{i=0}^{s} \partial^{i} F_{i}: F_{i} \in M_{N}[x]\right\} .
$$

Theorem 7.1. Given a weight $W$, if there exists an operator $C \in \mathcal{D}(W)$ such that $\Lambda_{n}(C)$ is a scalar matrix for every $n$ and $\Lambda_{k}(C)=\Lambda_{j}(C)$ only if $k=j$, then

$$
C_{\mathbf{D}}(C)=\mathcal{D}(W) .
$$

In particular, $C_{\mathbf{D}}(\mathcal{Z}(W))=\mathcal{D}(W)$.

Proof. Let $D \in C_{\mathbf{D}}(C)$, for a fixed $n \in \mathbb{N}_{0}$ we have

$$
P_{n} D=\sum_{j=0}^{K} A_{n, j} P_{j}
$$

with $\left\{A_{n, j}\right\}$ matrices and $K \in \mathbb{N}_{0}$. Since $D C=C D$, we have

$$
\sum_{j=0}^{K} A_{n, j} \Lambda_{j}(C) P_{j}=\sum_{j=0}^{K} \Lambda_{n}(C) A_{n, j} P_{j},
$$

thus

$$
A_{n, j} \Lambda_{j}(C)=\Lambda_{n}(C) A_{n, j}, \quad \text { for every } j .
$$

Therefore, $A_{n, j}=0$ for all $j \neq n$. Having then $P_{n} D=A_{n, n} P_{n}$, which implies $D \in \mathcal{D}(W)$. Therefore $C_{\mathbf{D}}(C) \subset \mathcal{D}(W)$.

Since $\Lambda_{n}(C)$ is a scalar matrix, it is clear that $C_{\mathbf{D}}(C) \supset \mathcal{D}(W)$. The first assertion follows. For the second assertion it is enough to observe that in general we have $C_{\mathbf{D}}(C) \supset C_{\mathbf{D}}(\mathcal{Z}(W)) \supset \mathcal{D}(W)$. 
With a similar proof, we can obtain the following result.

Theorem 7.2. Given a weight $W$, if for every $n$ there exists an operator $C_{n} \in \mathcal{Z}(W)$ such that $\Lambda_{k}\left(C_{n}\right)$ is a scalar matrix for every $k$ and $\Lambda_{k}\left(C_{n}\right)=\Lambda_{n}\left(C_{n}\right)$ only if $k=n$, then

$$
C_{\mathbf{D}}(\mathcal{Z}(W))=\mathcal{D}(W)
$$

Corollary 7.3. For the weight considered in the present paper and the weight considered in [Tir11] we have

$$
C_{\mathbf{D}}(\mathcal{Z}(W))=\mathcal{D}(W)
$$

\section{REFERENCES}

[AKdlR15] N. Aldenhoven, E. Koelink, and A. M. de los Ríos. Matrix-valued little q-Jacobi polynomials. J. Approx. Theory, 193:164-183, 2015.

[AS65] M. Abramowitz and I. Stegun. Handbook of Mathematical Functions with Formulas, Graphs, and Mathematical Tables. Dover, New York, 1965.

[BC23] J. L. Burchnall and T. W. Chaundy. Commutative ordinary differential operators. Proc. Lond. Math. Soc. (2), 21:420-440, 1923.

[BC28] J. L. Burchnall and T. W. Chaundy. Commutative ordinary differential operators. Proc. R. Soc. Lond., Ser. A, 118:557-583, 1928 .

[Boc29] S. Bochner. Über Sturm-Liouvillesche Polynomsysteme. Math. Z., 29:730-736, 1929.

[CdlI14] M. Cafasso and M. D. de la Iglesia. Non-commutative Painlevé equations and Hermite-type matrix orthogonal polynomials. Comm. Math. Phys., 326(2):559-583, 2014.

[CG05] M. M. Castro, , and F. A. Grünbaum. Orthogonal matrix polynomials satisfying first order differential equations: a collection of instructive examples. J. Nonlinear Math. Physics, 12(2):63-67, 2005.

[CG06] M. M. Castro, , and F. A. Grünbaum. The algebra of differential operators associated to a given family of matrix valued orthogonal polynomials: five instructive examples. Int. Math. Res. Not., 27(2):1-33, 2006.

[DdlI08a] A. J. Durán and M. D. de la Iglesia. Second-order differential operators having several families of orthogonal matrix polynomials as eigenfunctions. Int. Math. Res. Not. IMRN, pages Art. ID rnn 084, 24, 2008.

[DdlI08b] A. J. Durán and M.D. de la Iglesia. Some examples of orthogonal matrix polynomials satisfying odd order differential equations. Journal of Approximation Theory, 150(2):153-174, 2008.

[DG86] J. J. Duistermaat and F. A. Grünbaum. Differential equations in the spectral parameter. Comm. Math. Phys., 103(2):177-240, 1986.

[DG04] A. J. Durán and F. A. Grünbaum. Orthogonal matrix polynomials satisfying second-order differential equations. Int. Math. Res. Not., (10):461-484, 2004.

[DG05a] A. J. Durán and F. A. Grünbaum. A characterization for a class of weight matrices with orthogonal matrix polynomials satisfying second-order differential equations. Int. Math. Res. Not., 23:1371-1390, 2005.

[DG05b] A. J. Durán and F. A. Grünbaum. Orthogonal matrix polynomials, scalar-type Rodrigues' formulas and Pearson equations. J. Approx. Theory, 134(2):267-280, 2005.

[DG05c] A. J. Durán and F. A. Grünbaum. Structural formulas for orthogonal matrix polynomials satisfying second-order differential equations. I. Constr. Approx., 22(2):255-271, 2005.

[DG05d] A. J. Durán and F. A. Grünbaum. A survey on orthogonal matrix polynomials satisfying second order differential equations. J. Comput. Appl. Math., 178(1-2):169-190, 2005.

[dlI11] M. D. de la Iglesia. Some examples of matrix-valued orthogonal functions having a differential and an integral operator as eigenfunctions. J. Approx. Theory, 163(5):663-687, 2011.

[Dur97] A. J. Durán. Matrix inner product having a matrix symmetric second-order differential operator. Rocky Mt. J. Math., 27(2):585-600, 1997.

[GdlIMF11] F. A. Grünbaum, M. D. de la Iglesia, and Andrei Martínez-Finkelshtein. Properties of matrix orthogonal polynomials via their Riemann-Hilbert characterization. SIGMA Symmetry Integrability Geom. Methods Appl., 7:Paper 098, 31, 2011.

[GH97] F.Alberto Grünbaum and Luc Haine. A theorem of Bochner, revisited. In Algebraic aspects of integrable systems: in memory of Irene Dorfman, pages 143-172. Boston, MA: Birkhäuser, 1997.

[GPT01] F. A. Grünbaum, I. Pacharoni, and J. Tirao. A matrix-valued solution to Bochner's problem. J. Phys. A, 34(48):10647-10656, 2001.

[GPT02a] F. A. Grünbaum, I. Pacharoni, and J. Tirao. Matrix valued spherical functions associated to the complex projective plane. J. Funct. Anal., 188(2):350-441, 2002.

[GPT02b] F. A. Grünbaum, I. Pacharoni, and J. Tirao. Matrix valued spherical functions associated to the three dimensional hyperbolic space. Internat. J. Math., 13(7):727-784, 2002. 
[GPT03] F. A. Grünbaum, I. Pacharoni, and J. Tirao. Matrix valued orthogonal polynomials of the Jacobi type. Indag. Math. (N.S.), 14(3-4):353-366, 2003.

[GPT04] F. A. Grünbaum, I. Pacharoni, and J. Tirao. An invitation to matrix-valued spherical functions: Linearization of products in the case of complex projective space $p_{2}()$. Cambridge: Cambridge University Press, 2004.

[GPT05] F. A. Grünbaum, I. Pacharoni, and J. Tirao. Matrix valued orthogonal polynomials of Jacobi type: the role of group representation theory. Ann. Inst. Fourier (Grenoble), 55(6):2051-2068, 2005.

[GPZ15] F. A. Grünbaum, I. Pacharoni, and I. Zurrián. Time and band limiting for matrix valued functions, an example. SIGMA Symmetry Integrability Geom. Methods Appl., 11:Paper 044, 14, 2015.

[Grü03] F. A. Grünbaum. Matrix valued Jacobi polynomials. Bull. Sci. Math., 127(3):207-214, 2003.

[GT07] F. A. Grünbaum and J. Tirao. The algebra of differential operators associated to a weight matrix. Integral Equations Operator Theory, 58(4):449-475, 2007.

[Kre49] M. G. Krein. Infinite j-matrices and a matrix moment problem. Dokl. Akad. Nauk SSSR, 69(2):125-128, 1949.

[Kre71] M. G. Krein. Fundamental aspects of the representation theory of hermitian operators with deficiency index $(m, m)$. AMS Translations, series 2, 97:75-143, 1971.

[Kri81] I.M. Krichever. Algebraic curves and non-linear difference equations. Integrable systems, selected papers, Lond. Math. Soc. Lect. Note Ser. 60, 170-171, 1981.

[KV01] M. Kimura and P. Vanhaecke. Commuting matrix differential operators and loop algebras. Bulletin des Sciences Mathématiques, 125(5):407 - 428, 2001.

[KvPR12] E. Koelink, M. van Pruijssen, and P. Román. Matrix-valued orthogonal polynomials related to $(\mathrm{SU}(2) \times \mathrm{SU}(2)$, diag). Int. Math. Res. Not. IMRN, (24):5673-5730, 2012.

[KvPR13] E. Koelink, M. van Pruijssen, and P. Román. Matrix-valued orthogonal polynomials related to(SU $(2) \times \mathrm{SU}(2)$, diag) II. Publ. Res. Inst. Math. Sci., 49(2):271-312, 2013.

[Mir05] L. Miranian. Matrix-valued orthogonal polynomials on the real line: some extensions of the classical theory. J. Phys. A, 38(25):5731-5749, 2005.

[Mum77] D. Mumford. An algebro-geometric construction of commuting operators and of solutions to the Toda lattice equation, Korteweg de Vries equation and related non- linear equations. Proc. int. Symp. on algebraic geometry Kyoto 1977, 115-153, 1977.

[PR08] I. Pacharoni and P. Román. A sequence of matrix valued orthogonal polynomials associated to spherical functions. Constr. Approx., 28(2):127-147, 2008.

[PT07] I. Pacharoni and J. Tirao. Three term recursion relation for spherical functions associated to the complex hyperbolic plane. J. Lie Theory, 17(4):791-828, 2007.

[PZ15] I. Pacharoni and I. Zurrián. Matrix Gegenbauer polynomials: The $2 \times 2$ fundamental cases. Constructive Approximation, pages 1-19, 2015.

[Tak05] K. Takasaki. Tyurin parameters of commuting pairs and infinite dimensional grassmann manifold. arXiv:nlin/0505005, 2005.

[Tir77] J. Tirao. Spherical functions. Rev. Un. Mat. Argentina, 28:75-98, 1977.

[Tir03] J. Tirao. The matrix-valued hypergeometric equation. Proc. Natl. Acad. Sci. U.S.A., 100(14):8138-8141, 2003.

[Tir11] J. Tirao. The algebra of differential operators associated to a weight matrix: a first example. Polcino Milies, César (ed.), Groups, algebras and applications. XVIII Latin American algebra colloquium, São Pedro, Brazil, August 3-8, 2009. Proceedings. Providence, RI: American Mathematical Society (AMS). Contemporary Mathematics 537, 291-324, 2011.

[TZ14] J. A. Tirao and I. Zurrián. Spherical functions of fundamental $K$-types associated with the $n$-dimensional sphere. SIGMA, Symmetry Integrability Geom. Methods Appl., 10:paper 071, 41, 2014.

[TZ15] J. Tirao and I. Zurrián. Reducibility of Matrix Weights. ArXiv e-prints, January 2015.

[vPR14] M. van Pruijssen and P. Román. Matrix valued classical pairs related to compact Gelfand pairs of rank one. SIGMA Symmetry Integrability Geom. Methods Appl., 10:Paper 113, 28, 2014.

Facultad de Matemáticas, Pontificia Universidad Católica de Chile. Macul, Santiago, Chile.

E-mail address: zurrian@famaf.unc.edu.ar 